\title{
PREDICTIVE DISPLAYS FOR SURVEY VESSELS
}

\author{
Briana Sullivan, Colin Ware and Matthew Plumlee \\ Center for Coastal and Ocean Mapping \\ University of New Hampshire \\ Durham, New Hampshire
}

\begin{abstract}
Inexperienced helmsmen often oversteer because of the lag that occurs between changing the rudder angle and a change in the vessel's heading. Predictive displays are a common way of mitigating the effect of lag on human control. Accordingly we developed a predictive display to show the position and heading of a vessel a short time in the future. With this display, the helmsman's task becomes that of keeping the predictor on the planned path. In effect, the predictor is steered, not the vessel. Our predictive model was statistical and based on data gathered from a 40 foot survey vessel carrying out a variety of maneuvers while the position, heading, speed through the water and rudder angle were continuously recorded. The advantage of such a predictor is that it can, in principle, be generated automatically, without any need for a model of hull shape or vessel dynamics. We evaluated the predictor by having both experienced and inexperienced helmsmen steer a pre-defined figure-of-eight course. The results showed substantial reduction in cross track error for inexperienced participants to the point that their performance was indistinguishable from those that were more experienced.
\end{abstract}

\section{INTRODUCTION}

According to the boating reference Chapman Piloting: Seamanship and Small Boat Handling [Maloney, 2003] "As a vessel swings with a change in course, an inexperienced helmsman tends to allow her to swing too far, due to the momentum of the turn and the lag between him turning the wheel and the craft responding to it." This can result in wild deviations from the intended heading. Our study attempts to correct this error by using a predictive display that will eliminate the lag time in order to aid navigation as suggested by Wickens (2002). In a predictive display the helmsman of the vessel sees a computed image of where the vessel will be at a short time in the future based on the current control inputs. The helmsman's task is to steer the predictor not the vessel.

Other studies relating to lunar vehicles, aircrafts, and telemanipulations have shown success in decreasing error rates when such predictive displays are in use [Mathan, 1996; Bejczy, 1990; Sachs, 2000]. To deal with the time delay problem, dynamic virtual environments used as training tools for operators employ various control schemes [Sabater, 2005]. Predictors are also used on some high speed passenger-carrying vessels with the ability to "look ahead" from 30 to 120 seconds in order to contribute to vessel safety [Renilson, 1999]. Larger vessels also use different types of predictor displays such as container ships; however this type of predictor isn't used for real-time navigation.
We developed our own predictive model based on simple correlations between the vessel's present position, direction, and speed, and its previous rudder angle, position, direction, and speed as well as the direction and speed of the current acting on the vessel [Porathe, 2004]. This allows a model to be constructed automatically without a model of boat dynamics. Our simple and generic predictor is intended to be used on various types and sizes of vessels. It is the first time for this particular model to be evaluated in a formal study.

In our study, we investigated the use of a predictor by inexperienced helmsman steering a 40 foot survey vessel on a predefined course. The goal was to assess performance both with and without a predictive computer display. We used a constant velocity which Baldwin (1998) suggests will provide the best prediction results. The intended application of this study is to help novice helmsman accurately steer predefined survey lines.

\section{METHOD}

The hypothesis of our experiment was that novice helmsman would more accurately steer a vessel along a test course when shown a representation of the vessel 10 seconds into the future, than if they were simply shown a representation of the current position of the vessel.

The vessel was the R/V Coastal Surveyor, a 40 foot, 16 ton dedicated survey vessel. The ship's captain oversaw the safe steering of the vessel and was responsible for positioning it in the correct location and setting its speed 
(approximately 5 knots through the water) for the evaluations to take place.

For the experimental task, participants maneuvered the predefined course shown in Fig. 2, aided by a computer interface shown in Fig. 1. Participants were asked to monitor the computer interface and use the vessel's steering wheel to keep the proxy on the course. The average distance and difference in heading between the course and the path the vessel actually took as each subject steered was measured for later analysis.

The interface included an image of the bathymetry (a digital model of the seabed) over which the participants were maneuvering (Little Bay, NH) and the course that they were to follow. The interface also included a proxy, a small representation of the vessel.

The proxy was rendered as a flat, semitransparent boat icon as illustrated in Fig. 1. When the predictor was turned off, the proxy indicated the vessel's actual position and heading, updated twice per second from the vessel's onboard instruments. When the predictor was turned on, the proxy indicated where the vessel was expected to be. In this mode, the view on the screen shows the proxy's bow pointing to the top of the screen, regardless of where North really was, which makes the proxy appear as if it is static on the screen with the world (other objects on the screen) rotating about it. However, when the predictor was off, the boat icon did not statically point to the top of the screen, but instead changed heading with respect to the rudder angle.

The course was a circuit that took roughly 7 minutes to maneuver. It was approximately 650 meters in length and 200 meters wide with two turns, each about 100 meters in diameter. Each subject steered the course three times. The first circuit was a training session designed to introduce them to how the vessel handled and receive any basic driving advice the captain may share (for example, how to know what angle the rudder is at and how to use landmarks to navigate better). During training, half of the participants drove approximately half of the course with the predictor on and the other half with it turned off, and vice versa for the other half of the participants. The order the predictor was shown during training was the same order the predictor was shown for each subsequent full circuit on the course.

At the beginning of all the trials and again after each group of three or four sessions on the course, the current vector was entered into the system. These vectors were estimated by allowing the vessel to drift with the current and then checking the vessel speed via the instruments aboard. This process could have been automated if the vessel had been equipped with a sensor that calculated the speed over ground information. This value would then automatically feed the predictive display instead of having to do periodic drift tests. Automation in this way would make the input values more accurate throughout the testing time since it would be continuously updating real time values.

The experiment was carried out over the course of 11 days between Sept. 16, 2005 and Nov. 7, 2005 with testing times between 7:35am and 5:55pm depending on the tide schedule for the given day. The weather varied from warmer, clear, calm days to cool, damp, windy days. None of these external variations were factored into the calculations for the predictor.

\section{Participants}

A total of 25 people, all affiliated with the University of New Hampshire (UNH), participated in this study. They were given a short survey to fill out afterward in order to categorize their prior boating experience.

Originally there were 5 categories for participants to choose from to describe their level of boating experience: 1) have never been on a vessel (this didn't apply to any of the participants), 2) been on a vessel less than 100 hours but had never steered it (this applied to 10 participants), 3) steered a vessel less than one hour (this applied to 8 participants), 4) steered a vessel less than 100 hours (but more than 1 hour this applied to 3 participants), 5) steered a vessel more than 100 hours (this applied to 4 participants). Therefore the first 3 categories (those with less than one hour steering a vessel) were combined into the novice group (18 people) and the remaining categories (those with more than one hour) were considered experts ( 7 people).

\section{The Predictor Model}

We constructed the model underlying the predictor by running linear regressions on data collected prior to the experiment. Basic inputs to the model include the rudder angle, course over ground, and heading. Outputs include changes in heading and course over ground at every second up to 10 seconds into the future. Data was recorded from the $\mathrm{POS} / \mathrm{MV}$ and autopilot instruments aboard the vessel as the vessel traced out straight lines, a few turns, and a weaving pattern, all at speeds in the range chosen for the experiment (between 1200 and 1500 RPMs). The data was collected at high tide to minimize the effects of current.

The following outputs were generated from the regression model. The angular velocity of vessel heading was computed as: $\mathrm{Hv}_{t}=$ Heading $_{t}-$ Heading $_{(t-1)}$. The course over ground at a given time $t\left(\mathrm{COG}_{t}\right)$ was computed from changes in recorded position. From this, the angular change per second was calculated: $\mathrm{COGv}_{t}=\mathrm{COG}_{t}-\mathrm{COG}_{(t-1)}$. To support the regression, historical quantities for rudder information Rudder $_{(t-1)}$ and $\operatorname{Rudder}_{(t-2)}$ ) were generated, as well as changes between the "current" and "future" quantities of course over ground and of heading $\left(\Delta \mathrm{COG}_{(t+1)}\right.$ through $\Delta \mathrm{COG}_{(t+10)}$ and $\Delta$ Heading $_{(t+1)}$ through $\Delta$ Heading $\left._{(t+10)}\right)$. With these quantities in place, the SPSS statistical package was used to run the twenty linear regressions we needed:

$$
\begin{aligned}
& \Delta \text { Heading }_{(t+\Delta t)}=a \cdot \text { Rudder }_{t}+b \cdot \text { Rudder }_{(t-1)}+c \cdot \\
& \text { Rudder }_{(t-2)}+d \cdot H v_{t}+K \text {, } \\
& \text { and } \\
& \Delta C O G_{(t+\Delta t)}=a \cdot \text { Rudder }_{t}+b \cdot \text { Rudder }_{(t-1)}+c \cdot \text { Rudder }_{(t-2)} \\
& +d \cdot H v_{t}+f \cdot C O G v_{t}+K \text {, } \\
& \text { where } 1 \leq \Delta t \leq 10 \text {. }
\end{aligned}
$$


In still water, the regression equations could be applied to incoming sensor data simply by using the last few seconds of rudder information and calculating the change over the last second in heading and course over ground. However, our experiments were carried out in a significant current. To account for this, we estimated the vessel's speed and course through water by subtracting an estimated current vector. This had the effect of transforming the prediction situation into one in which the still-water assumption was valid. We then applied the regression equations and added the current vector back onto the predicted values, thereby transforming them back to the real-world situation.

\section{RESULTS}

The results of the experiment are summarized in Figure 3 , where points to the right of the diagonal line indicate improvement in either cross-track-error or heading error. Figure 2 illustrates the actual paths followed by a particular novice subject (left pair) and a particular experienced subject (right pair). Within each pair, the distance (blue lines) between the steered path (red curve) and the intended course (implied) is shown when the predictor was off (left image) and on (right image).

An analysis of variance was performed to establish whether use of the predictor and a subject's level of experience contributed to the average distance a subject steered from the intended course. The analysis revealed that use of the predictor had a significant effect $(\mathbf{F}(1,46)=$ $11.12 ; \mathbf{p}<0.005)$, and that subject experience was marginally significant $(\mathbf{F}(1,46)=4.07 ; \mathbf{p}<0.05)$. The interaction between use of the predictor and subject experience was also significant $(\mathbf{F}(1,46)=5.75 ; \mathbf{p}<0.05)$. An analysis of variance was also performed to determine the effect the same factors had on average angular difference between the steered heading and the heading prescribed for the nearest point on the intended course. The analysis revealed that use of the predictor was the only factor to have a significant effect $(\mathbf{F}(1,46)=6.55 ; \mathbf{p}<0.05)$. Finally an analysis of variance was performed to determine whether or not experience level was a significant factor in cross-trackdistance when the predictor was on. The analysis showed that experience level was not a significant factor.

\section{DISCUSSION}

We have created a predictive display that is based on statistical analysis of time series data acquired from an instrumented 40 foot survey vessel. The predictor takes in rudder angle, position and heading measurements and produces a predicted position and heading, relative to the vessel, a few seconds into the future. The advantage of this type of predictor is that no formal model of vessel dynamics is required. Such a predictor could, in principle, be generated automatically for any suitably instrumented boat. In the future, it is possible that predictive models could automatically adapt, based on local changes in the sensor input and the statistical model itself could be refined to include measurements from water depth and wind loading sensors.

Results from our evaluation show that the predictor helps novices reduce their average distance from the intended track enormously to the point that their cross track error is statistically indistinguishable from that of an experienced helmsman. This is encouraging, although a number of issues must be addressed, before deployment could be contemplated. One of the potential problems, is that in order to take advantage of the predictor, the helmsman may spend less time looking out of the window, with a corresponding loss in overall situational awareness.

We believe that this kind of predictor is suitable for vessels in the $20-120 \mathrm{ft}$ range where steering is done by means of closed loop control with the human operator in the loop. For vessels smaller than this the effect of rudder changes is much more immediate and so over steering is less of a problem. For larger vessels steering is generally done by setting a heading in an open loop fashion with respect to the helmsman and this concept would be less applicable.

\section{Acknowledgements}

Support for this project was provided by NOAA Grant NA170G2285. We are grateful for technical assistance from Roland Arsenault. We are also grateful to Ben Smith the skipper of the Coastal Surveyor for his calm assistance.

\section{REFERENCES}

Baldwin, J., Basu, A., and Zhang, H. (1998). Predictive windows for delay compensation in telepresence applications. IEEE International Conference on Robotics and Automation (ICRA), Leuven, Belgium, IEEE Robotics and Automation Society. 2884-2889.

Bejczy, A. K., Basu, W. S., and Venema, S. C. (1990). The Phantom Robot: Predictive displays for teleoperation with time delay. IEEE International Conference on Robotics and Automation (ICRA'90), Cincinnati, $\mathrm{OH}$. 546-551.

Maloney, E. S. (2003). Chapman Piloting \& Seamanship, Hearst Books. pg. 175.

Mathan, S., Hyndman, A., Fischer, K., Blatz, J., and Brams, D. (1996). Efficacy of a Predictive Display, Steering Device, and Vehicle Body Representation in the Operation of a Lunar Vehicle. Proceedings ACM Conference on Computer Human Interaction (CHI Conference Companion). 71-71.

Porathe, T. (2004). Visualizing the Decision Space of a Ship's Maneuverability in a Real-Time 3-D Nautical Chart. The NATO Research and Technology Organisation Workshop - Visualisation and the Common Operational Picture (VizCOP), Toronto, Canada. 159-164.

Renilson, M. (1999). The Specialist Committee on Safety of High Speed Marine Vehicles (HSMV). International Towing Tank Conference, Seoul and Shanghai, 22nd ITTC Specialist Committee. 
Sabater, J. M., Azorin, J. M., Reinoso, O., Neco, R. P., and Garcia, N. M. (2005). "Dynamic virtual environment to test teleoperated systems with time delay communications." Journal of Robotic Systems 22(4): 167-181.
Sachs, G. (2000). "Perspective Predictor/Flight-Path Display with Minimum Pilot Compensation." Journal of Guidance, Control, and Dynamics, 23(3), 494-501.

Wickens, C. D. (2002). "Situation Awareness and Workload in Aviation." Current Directions in Psychological Science 11(4): 128 - 133.

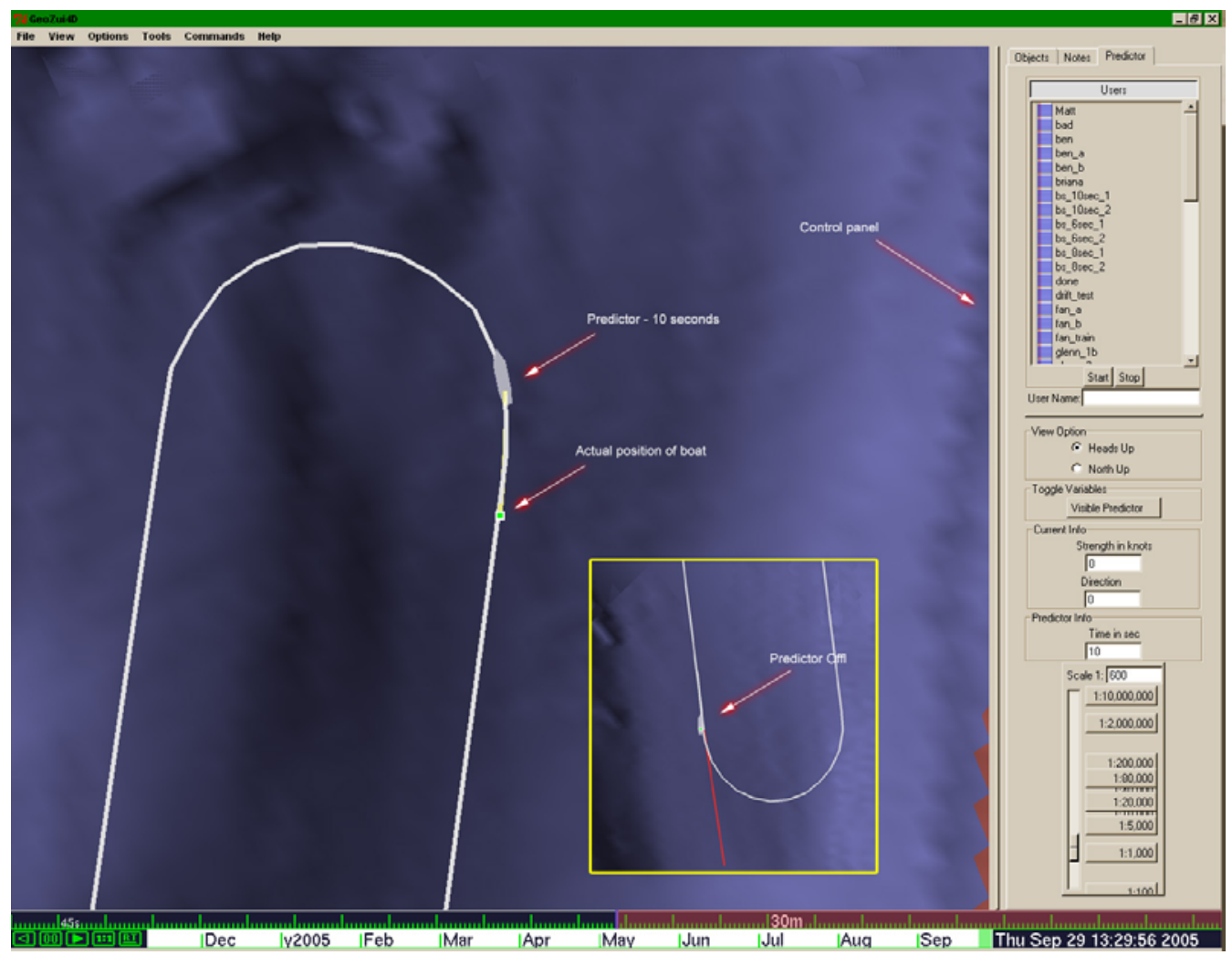

Figure 1. User view with predictor on, and off (inset).

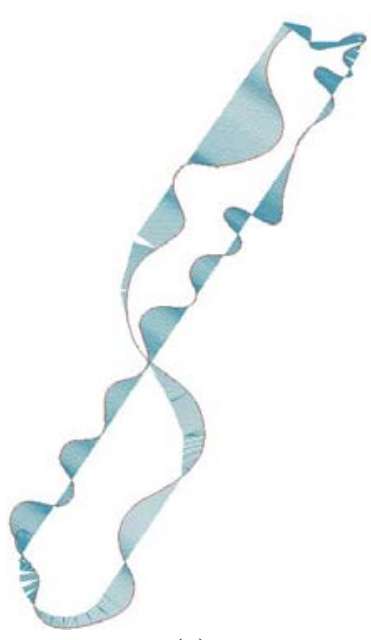

(a)

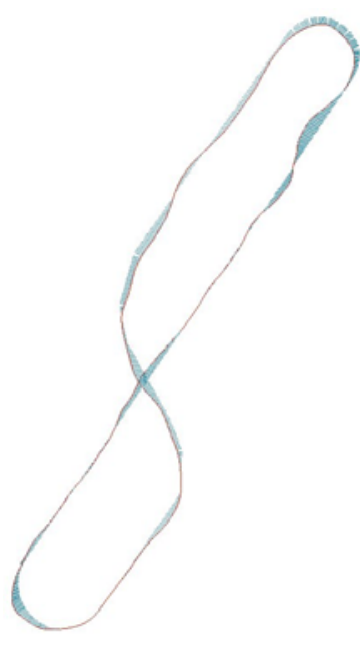

(b)

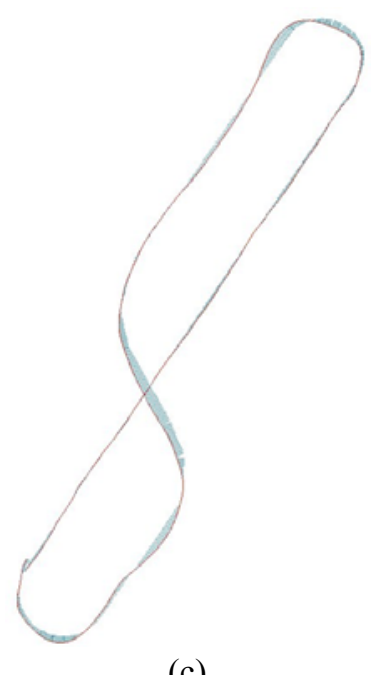

(c)

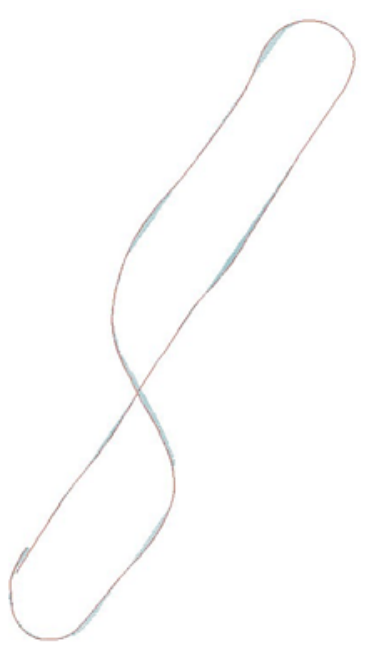

(d)

Figure 2. Samples of cross-track distance for a novice participant with the predictor (a) off and (b) on, and for an experienced participant (c) off and (d) on. 

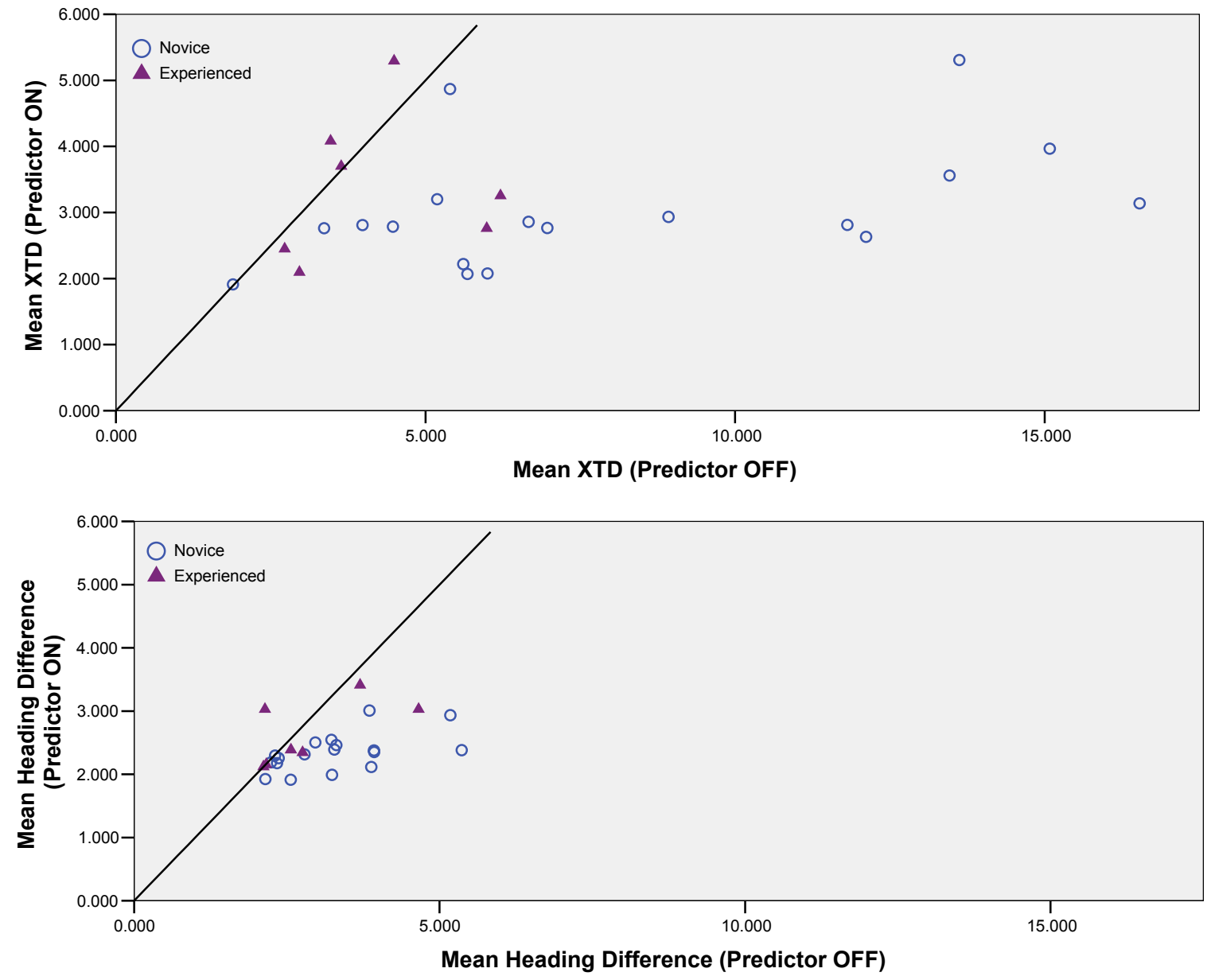

Figure 3. Plots of how well each participant steered the intended course in terms of cross-track distance (XTD) (top) and difference in heading (bottom). The diagonal line in each plot indicates a "no-change" threshold; points below and to the right represent participants who performed better with the predictor. 